\title{
Discussion on Application for Interior Space Design and the Application of Interior Design Style
}

\author{
Xiaodong Zhang \\ Institute of Environmental art design, Hebei Institute of Fine Art, Shijiazhuang hebei, 050700, China
}

Keywords: interior design, psychological space, design style

\begin{abstract}
Interior design is the essence of the interior space design, including physical space design and psychological space design two levels. Interior design style is an important issue for interior designers and owners. Through analysis and Research on the relationships between design and interior design style of interior space, summed up the "interior space design to create a style of interior design" and "interior design style shape the psychological space two obvious characteristics.
\end{abstract}

\section{Interior Space Design and Its Content}

The Meaning of Interior Space Design. Interior space design is a creative activity of human transformation of living space, through the interior space design can make human living environment become more comfortable [1]. Although the "interior space design" theory is not a long time, but has been constantly being human practice. Since ancient times, human beings have been in the pursuit of the security of living space.

Full, comfortable and beautiful. People in the primitive society in the cave laying leaves, weeds and skins and in the walls of the cave with color drawing lines and patterns, and so on for living space transformation, which all belong to the indoor space design. Interior space design is not only focused on solving the relationship between people and nature, but also more and more focus on solving the problem of the relationship between people and society, so that people in the community better work and life.

Content of Interior Space Design. Interior space design is often based on the interface design, color design and material design, etc.. The interior space interface is the enclosure of the interior space, which consists of the top surface, the bottom surface and the side surface. Through the space interface design, we can create a sense of depth, space and sense of scale, and make the space with a certain function and orientation.

The materials used in the design of space are rich and varied, and the processing technique and technology are more and more diverse. According to the effect of the design, the interior space design can be divided into two categories, physical space design and psychological space design.

Physical Space Design. In order to consider the indoor function as the main, the design of the physical environment is called the indoor physical space design. Using walls and partitions for indoor functional space division, TV background wall modeling design, top surface ceiling practices are all belong to the scope of physical space design. Special sound environment, light environment, thermal environment, texture and texture of objects in the interior space are the essence of the physical environment, which can be perceived by people through the visual, tactile, auditory, [2]. Physical space design technique is very rich, plants use spatial organization design, interface design and decoration with fabric soft, use of lighting, lighting design, furniture for use in functional organization and storage design, space division with carpet tiles marble. The use of new technologies, new processes and reasonable organization of these physical environment, in order to 
make people better use of indoor space, in order to allow indoor space to play their own role in regulating the relationship between people and nature, people and society. The physical space design is the expression form and the carrier of the psychological space design, without the support of the physical space design.

Psychological Space Design. Begin in the physical space of the interior design, its focus is the design of psychological space. Mental space.

Design more emphasis on people's spiritual world and the design of psychological feelings. Mental space design needs to have its material carrier, often with the aid of physical space design to reflect. Figure 1 based on the wall and partition the space segmentation is a typical physical space design, but because of space to leave dining area and living room to receive visitors area with different functions of psychological impression. Figure 2 use of lighting, the space partition design is in the physical space design clever the psychological space design [3], although there is no clear wall separated, but light and furniture has made people in the same room clearly feel their location and status. At the same time, an office is divided into two different functional areas, focusing on the identity and status of the display and shape the authority of the leadership of the image. In addition, in this not spacious interior space in the design of a mirror can accurately reflect the interior scenery, can make people have a more open interior space of psychological impression. All of these belong to the design of psychological space, through the method of physical space design, attention to the indoor space of the building is an important part of interior design.

\section{The Generation and Function of Interior Design Style}

The Formation of Interior Design Style. The interior design of a region, an era or a relatively fixed stage, all of which are affected by the political, economic, cultural and other factors in the same social background, reflect some common characteristics. In long term

In practice, people continue to summarize the methods of space design, in different periods, different regions, different cultural backgrounds

Under the influence of gradually formed a relatively fixed approach. For example, by the authority of the supreme authority of the ancient Egyptian pharaoh

In ancient Egypt, the influence of the treatment on the background of ancient Egypt, which reflected the class difference and the special way of life. Interior design [4]. With the above four is corresponding to the distinctive features of the interior design style were known as the ancient Greek style interior design, interior design style of ancient Rome, medieval European style interior design and the Renaissance style interior design, and are collectively referred to as the classical style of interior design. Again, under the big background of the industrial revolution, to production machine instead of manual production and by the German Bauhaus promote and the development of modernist style prevalent in many countries in the world, but also the social background decided to design style of typical cases. Types of around the world in each period of the interior design style is very rich, when we to each kind of interior design style scrutiny, we can found that the formation and development of each kind of interior design style is with social background have close relationship. It can be said that the social background determines the style of interior design.

The Relationship Between Design Style and Social Background. The formation of interior design style is the result of complex social background. Different social backgrounds, interior style also reflects their different characteristics, all of the interior design style has its unique social background. The naming of interior design style is decided by the social background. The social background of a region is different, it is possible to produce a regional named interior design style, such as: Nordic style, American style, style of Japanese and Korean, Chinese style, etc. The social 
background of an era is different, it is possible to produce the interior design style named by the times, such as: classical style, modern style, the style of the Han Dynasty, the style of the Qing Dynasty, etc.. Different ethnic groups of different social backgrounds, it is possible to produce the national name of the interior style, such as: Korean style, Mongolia style, etc.. Some style of interior design by political, religious, and cultural influence was once popular in many countries and continued the development of more than a century, such as Baroque interior, Rococo interior. But no matter how named, the interior design style naming always reflects its relationship with the social background is inseparable. Because the interior design style is produced in a certain social context and has its social background in line with the common characteristics, which can continue to reflect the social background of the formation of its style.

Design Style of the Psychological Role. A certain style of interior design can make people have a rich association with the specific social background. Indoor Design style reflects the characteristics of the times and cultural characteristics, is used to beautify the life of an important home Volume. The interior design of traditional Japanese style use "sitting and sat on the floor" use low furniture, With the entry of foreign culture, the new interior design and furniture design style has been accepted by the Japanese. But in the day Style bedroom in the traditional "and the design of the wind" or modern "foreign design", most people will choose to leave at least one use of tatami typical traditional style "and". The purpose is to do so. Retain the nation's traditional culture and ethnic custom kneeling tea. Visible interior design style can be inherited Cultural tradition, which has the effect of spiritual enlightenment. Different styles have different characteristics, but also can give people different Psychological hint and psychological inspiration.

Interior design style to the traditional China as an example: the Han Dynasty inherited the characteristics of the Qin Dynasty "brilliant grand", advocating a big beauty, interior design style of Han Dynasty to leave the atmosphere impression; the Tang Dynasty political power, economic development, interior design has the characteristics of fresh and lively, Hua Guifu Li, Tang Dynasty style of interior design can be used to produce elegant noble mental hint; Song Dynasty interior design style and elegant qualities, reminiscent of literati temperament, is to create a literary temperament room design preferred; interior design of Ming style furniture and Ming Dynasty style reflects the characteristics of "Jane thick elegance", this room design becomes the majority choose. And reflected in the Chinese feudal society during the heyday of the Qing Dynasty style interior design, fade deliberately decorative and artificial ingredients, often be used for dining space in interior design. It can be seen that the design style has its psychological implications and inspiration.

The interior design of the new Chinese style is based on the different styles of different dynasties, and is also active in the extraction of elements from the traditional culture of a certain area of the traditional architecture and the traditional culture of a minority. when designers use China Jiangnan traditional architectural style interior design, let a person naturally think of Jiangnan region of rivers and lakes in the humid climate and literati temperament, can reflect the Jiangnan region of rivers and lakes, the quiet and elegant atmosphere. Can reflect the master of the house literati temperament and elegant style.

\section{The Relationship Between Interior Design and Interior Design Style}

Mental space design is an important part of the interior space design. Interior design style is derived from the accumulation of culture and its refinement, interior design style has a form of characteristics and is not equivalent to form. Interior design style from the beginning, it is destined to be able to form a symbolic language to reflect the culture, and has obvious psychological 
implications and inspiration. Interior space design and interior design style of the relationship between the performance of "space design to create an interior design style" and "interior design style psychological space" these two aspects of the characteristics.

Space Design to Create a Style of Interior Design. Interior design style is a reflection of the social background, is a regional or a representative of the era of interior design. Space design is a creative design activity that needs to be combined with the needs of the owners and the characteristics of the building. Interior design is the process of the original interior space interface according to a certain law of the top, bottom and side of the transformation of design thinking and performance. Designers tend to analyze and define the style of the room first, on the basis of the definition of style to consider whether the indoor physical space design can be matched with style. Through the indoor interface and indoor sound, light, heat, electricity, water, warm and so on the overall design of the environment to create the entire interior space design style. Design style how to be reflected in a room all depends on the interior of the physical space design, the appropriate physical space design can be very good to show the full style of interior design features. Feng Xiujuan designer in New Chinese style interior design using traditional Chinese swastika pattern, fret, $\mathrm{Fu}$ lines and longevity lines patterns of TV setting wall and furniture design. From the Ming Dynasty style furniture chair circle design with Chinese characteristics back sofa. Furniture modeling and color applications highlight the Oriental style, clever use of decorative pillars in the background of the sofa to create the traditional architectural tradition of Liang Zhu. These specific methods of space design for the interior space to create a Chinese style atmosphere played an important role.

Interior Design Style to Shape the Psychological Space. Interior design style is a summary of the regularity and the times of the interior design. It comes from the cultural and social background of a certain era. It doesn't make sense to prefer the traditional style of interior design for the elderly. Middle aged and older people are generally more extensive than young people experience, they have their own understanding of life. For the rich experience of middle-aged and elderly people in the interior design, if the use of traditional style is more easily because of the traditional style of interior design and cause their cultural heritage. When we are in a foreign country or come to a foreign embassy, we often feel the foreign culture and the national culture which we have long formed.

Interior design for the Chinese people living in a foreign country, if appropriate for the construction of "China Wind" can remind the owner of homesickness feelings, so they got back to the house there is a real feeling of home. And if the Embassy in the country to make full use of the traditional style of the design elements of interior design, it is more likely to make people feel the presence of their own culture. All of the interior design style has certain cultural foundation, the use of interior design style is the essence of the special culture by is through in interior design using appropriate style reflects the culture shaping the psychological space.

\section{Soft Decoration}

Interior decoration can be divided into two parts, "soft" and "hard". The so-called "hard" decoration mainly refers to the wall, the ground, the top surface of the fixed decorative [5], such as the ground floor, top floor ceiling, etc.. These decorations are the main body of interior decoration, which plays a structural role. Soft decoration, the original view that mainly in the indoor fabric based soft materials for decoration. Now this define already was broken, in addition to fixed decoration, as long as it is to improve indoor function, contrast the indoor atmosphere, reflect the decoration of indoor culture, can be viewed as soft decoration. Hard decoration and decorative indoor soft 
adornment is equally important, imagine a space if only hard decoration, is rather too chilled, lack of affinity. And hard decoration is the main interior decoration, soft decoration is based on the hard decoration of the decoration, they are more like To Waba's relationship, are indispensable.

\section{Interior Design Style}

With a variety of cultural communication is increasingly close, between the style of modern interior design has no clear signs, there is no a style is another style replaced, "style" more Hou may is known as "the fashion". Although a variety of style contests, but in the design to be more or less applied to soft decoration. This is determined by its own characteristics, because soft decorative category has been expanded to indoor space all can move in the decoration, and are different, involving a wide range of designers are very easy to pick out the elements for the mutual collocation design style. At the same time, due to the flexible and easy to replace the soft decoration features, compared to the fixed space in the decoration, you want to change the design style, changing the space of soft decoration is more economical and convenient. Such as Tadao Ando's Church, although indoor space almost without any decoration, but the quaint wooden tables and chairs as a space of soft decoration, it and the whole space collocation is very harmonious, make the space appears more solemn and holy. And the light and shadow on the wall can also be seen as part of the soft decoration, because with the changes in the intensity of the light, the walls of light and shadow effects also produce undulating changes, the atmosphere of the indoor space plays an important role in regulating the.

\section{Soft Decoration in the Interior Design of the Use of Style}

Decorative Elements of Their Own Style. Is soft decoration, many soft decoration itself is a work of art, with a significant style features, such as Chinese style furniture, classical European style sofa shows obvious regional style with geometric patterns of carpet embodiment of modern style. These soft decoration in decorative symbols, decorative patterns, form, color, with a distinctive style characteristics, once placed in the room, it will make the entire space of the corresponding characteristics of the style. In the face of a no personality and style to speak of the space, to does not change under the premise of the decoration pattern, transforming it into the obvious style of the design space using the shortest period of time, you can make full use of soft decoration style, try to change the entire space style. In the restaurant, designers in the table using the carved with classical pattern of stone as a partition, originally simple space immediately vivid "of the design details of the decision", many space soft adornment to become a bright spot in the space, which is why designers to focus on the interior soft decoration.

Decorative Elements to Highlight the Overall Style of the Repeat. Modern interior design, pay attention to the integrity of the design, not only between the interior of each surface form, material, color to coordinate with each other, and soft interior decoration to have integrity and coherence. This will help to form a unified visual experience, so as to create the overall style. To achieve this effect, the most convenient way is to use the same decorative elements with the soft decoration. For example, you want to create a garden style space, in the design of soft decoration can choose to have the same pattern of the furniture and fabric, floral pattern itself reflect the pastoral style, such a pattern in the space repeated emphasizes more on the style of their own characteristics, so that the whole space is more holistic.

Mutual Collocation Create a Space Style. Compared with the use of the same elements with the soft decoration, a variety of soft decoration between collocation is clearly more commonly used. 
If you want to reflect the overall, coordinated the design style, in a variety of soft decoration to match each other there are at least two ways: using the same style soft decorative; or different styles of soft decoration through coordinated design methods put together.

It is one of the most popular methods for designers to use the same style of soft decoration in the space to reflect the interior style. designers want to create a Chinese style space, so use the sofa and coffee table is bamboo furniture with Chinese style, also with a picture of a small freehand brushwork in traditional Chinese painting lotus as the sofa background. This, of course, is a safe practice. The same style soft decorative collocation make whole and interior space style with obvious Chinese flavor.

But in the same space, the same style elements of the decoration used too much, it will inevitably give people the feeling of rigid. Some designers bold use of different styles of soft decoration, put it together, this is a test of the ability of the designers work. with Chinese meaning flower Windows partition and full of modern furniture, colorful paintings placed in the same space, these decorative elements individually different style, but in this space is very harmonious.

\section{The Interior Design of Minimalism}

For decades, minimalism has influenced the development trend of construction industry and decoration industry, the art world is mixed. When it comes to minimalism, he had mentioned - mid twentieth Century the most famous architectural master of Ludwig Mies van der rohe. He put forward the "less is more" (less is more) important ideas and themes, creating a minimalist precedent in the field of construction and decoration. He advocated the form of simple, functional and rational combination of design concept, put forward a new concept of "mobile space", abandon the cumbersome luxury of the design style. Every detail of his work is simplified to an extreme state that can no longer be simplified, and even the structure is exposed to a part of the art as a work of art. This is not what he sought was crudely made, reflects the quality, material space.

With the development of society, housing conditions continue to improve, more and more people are not satisfied with the function of the house is only to meet the living behavior. Modern people from the traditional family living mode is more and more far, reflecting the self value, highlighting the personality of the small family in recent years by many people sought after, especially by young people. These people no longer mind whether the spacious, convenient transportation, residential planning is reasonable, they care about is the interior design must be concise, lively, full of fashion sense. Minimalist design style just cater to the needs, it creates a quiet and calm of the space environment is a manifestation of the high-quality, seems to have become very popular nowadays is a design style.

Minimalist interior design can better cater to the potential requirements of space, although the principle of reduce the infinite, but with a highly rational attitude and principles. Layout, furniture display space and accessories are measured by. It is possible to use the elements of space itself as a decoration, such as structure, light and so on. Before people always to the decoration of the entire space rich, minimalism design concept to people's ideological liberation. It makes extensive use of simple lines, the basic geometry, pure color obtained high grade in a smooth, transparent, elegant, fashion design.

Furniture Design. In order to avoid the space is too monotonous, so the furniture in the minimalist interior design becomes very important. The use of a large number of single form of furniture, it should reflect the layout of the overall sense of unity, so that the design and placement of the furniture and the existence of the environment of integration. To fully consider the function of furniture in the design, exterior styling keep it simple, reasonable design of the storage space inside, 
especially bedroom closet, kitchen condole ark, to try to cater to the user's habits and habits. Most of the use of black and white color of three colors, the main color of the main color, simple but elegant. In the choice of materials comply with "reduction" principle, to avoid the collision of a variety of materials and complex process and decorative elements around less, pale line elements corresponding to the reduced. Only in this way can make furnishings more prominent, more rich modern sense, highlighting the inherent appeal, to create a free from vulgarity indoor environment. To some extent, this design can make people feel relaxed, calm and peaceful, and can meet the needs of modern people.

Space Layout. Minimalist design room layout with great propriety, not excessive, from the basic elements, including space and its edge and necessary furniture decoration. The entire space to choose a single color, grey or bright colours, the design according to "reduction, reduction, to reduce" principle reflects the charm of deep space, strive to use with a small amount of decoration means to create elegant home environment. At the same time also reflected in the interior decoration of the fine selection. Simplify the interior decoration, so that the focus of the space of vitality, can make people in the busy daily life to find a relaxed and free visual leisure space. In addition to providing the only necessities, no longer place other unnecessary things. The purpose of design is to make the entire space looks simple and connotation, for the selection of interior furnishings is to elaborate, it is able to render unique indoor atmosphere, to highlight the theme, and can reflect master individual character and grade.

Application of Materials and Colors. Minimalist interior design attaches great importance to the various materials and color the selection and application of, the abundant expressive force of materials can be achieved through the combination of a variety of building materials. Its design style is not suitable for the use of a large area of soft decoration or fabric, so the wall is one of the important elements of the embodiment of the style. Paint, brick, latex paint, mirror, steel and other commonly used wall decoration materials, in order to form a pure body mass visual impact, usually a large area of the use of the same material, more likely to be noticeable in the environment. In addition, the transparent space is in line with the public aesthetic, you can use some modern materials to achieve the effect of transparency. Such as the film is like leather has good transparency, can meet the requirements of indoor lighting, from outside to inside the transparent or from the inside outward diffusion of light, the light between the material back and forth reflection, refraction, resulting in wonderfully rich of integrated light effect, building the permeability and light can be realized, and the space itself with a sense of high quality, make people enjoy the space the rich connotation. Material on the ground can not be ignored, most of the use of monochrome stone or wood, and the wall of the material and color Coordination. Also although minimalist interior design in the accessories, but the appropriate selection of a small amount of accessories can also play a role in the finishing touch. For example, in the cold tone of the solid wood floor placed a soft texture of the carpet, the room will be filled with affinity. Curtain material should be chosen plain blinds or translucent gauze qualitative curtain, the curtain can increase the sense of space of the room, and also is more convenient and natural light to enter. Soft materials commonly used in interior design of minimalism with velvet, velvet, linen fiber, leather, silk, cotton, etc.. Try to use floral patterns or plain no pattern fabric, even if there are patterns also should not be too exaggerated, try to highlight the fabric texture and improve the space of the whole class. Use the area also according to the size of the space, furniture design and other accessories to use, so as to make the space is not too light. The light colored color, such as black, white and grey. Or in a few local use exaggerated high chroma color or green plants to be decorated, the space given the strong sense of art, full of vitality.

Lighting Design. Lighting design is very important in interior design. Light can bring a wealth 
of visual experience, and even the selection of materials and furniture design can not be done. Everyone on the light sensitive degree and the affection degree different, some people like to stay in a well lit place, some people like to stay in the dark, and minimalist of natural light has a special strong preference. Light in the space shuttle freely, unrestrained, away all empty and dull, directly to get close to nature, light with the change of day and night and changing, emitting a halo of different angle and different strength, reach their pursuit of beautiful and comfortable feeling of space.

The key of minimalist concept to the modern interior design in the design is the use of modern design techniques and concise form. In the space layout, the pursuit of pure space, emphasizing the function of space, that is, with the least amount of material to create the most decorated space. In terms of color, hue selection of subtle or exaggerated contrast is a minimalist ideas embodied, solid black, white, grey, the use of a large area, local ornament bright high chroma color embellishment, prominent space to bring people's visual impact. In the lighting design, the use of complex lighting to create brilliant lighting effects, clever use of natural light to achieve rich space, so that the space becomes vivid.

Minimalism to us that without redundant decoration, using extremely simple, geometric form, using a simple method, can create a dynamic, high quality work.

Through the analysis of the meaning and content of interior space design and the formation of interior design style and psychological function, the relationship between interior design and interior design style is analyzed and studied. Interior design and interior design style to the human mind as a bridge to produce a subtle link, reflected as "through the space design two obvious characteristics of "building interior design style" and "interior design style". Palm grasp these two characteristics for the use of interior design style interior design has a very good inspiration. In designers should pay more attention to the social background and the reflection of a design style. Culture, so that the interior design is more reasonable. Only by fully considering the psychological function of the inherent culture in the design style in order to better carry out the interior space design.

Under the influence of gradually formed a relatively fixed approach. For example, by the authority of the supreme authority of the ancient Egyptian pharaoh in ancient Egypt, the influence of the treatment on the background of ancient Egypt, which reflected the class difference and the special way of life. Interior design. With the above four is corresponding to the distinctive features of the interior design style were known as the ancient Greek style interior design, interior design style of ancient Rome, medieval European style interior design and the Renaissance style interior design, and are collectively referred to as the classical style of interior design. Again, under the big background of the industrial revolution, to production machine instead of manual production and by the German Bauhaus promote and the development of modernist style prevalent in many countries in the world, but also the social background decided to design style of typical cases. Types of around the world in each period of the interior design style is very rich, when we to each kind of interior design style scrutiny, we can found that the formation and development of each kind of interior design style is with social background have close relationship. It can be said that the social background determines the style of interior design.

The formation of interior design style is the result of complex social background. Different social backgrounds, interior style also reflects their different characteristics, all of the interior design style has its unique social background. The naming of interior design style is decided by the social background. The social background of a region is different, it is possible to produce a regional named interior design style, such as: Nordic style, American style, style of Japanese and Korean, 
Chinese style, etc. The social background of an era is different, it is possible to produce the interior design style named by the times, such as: classical style, modern style, the style of the Han Dynasty, the style of the Qing Dynasty, etc.. Different ethnic groups of different social backgrounds, it is possible to produce the national name of the interior style, such as: Korean style, Mongolia style, etc.. Some style of interior design by political, religious, and cultural influence was once popular in many countries and continued the development of more than a century, such as Baroque interior, Rococo interior. But no matter how named, the interior design style naming always reflects its relationship with the social background is inseparable. Because the interior design style is produced in a certain social context and has its social background in line with the common characteristics, which can continue to reflect the social background of the formation of its style.

A certain style of interior design can make people have a rich association with the specific social background. Indoor Design style reflects the characteristics of the times and cultural characteristics, is used to beautify the life of an important home Volume. The interior design of traditional Japanese style use "sitting and sat on the floor" use low furniture, With the entry of foreign culture, the new interior design and furniture design style has been accepted by the Japanese. But in the day Style bedroom in the traditional "and the design of the wind" or modern "foreign design", most people will choose to leave at least one use of tatami typical traditional style "and". The purpose is to do so. Retain the nation's traditional culture and ethnic custom kneeling tea. Visible interior design style can be inherited Cultural tradition, which has the effect of spiritual enlightenment. Different styles have different characteristics, but also can give people different Psychological hint and psychological inspiration.

\section{References}

[1] Ma Jian-mei. Environmental art course teaching mode reform of professional landscape design [J]. Anhui Agricultural Science. 2011 (31)

[2] Tang Jun. The development of modern landscape design present situation and trend analysis [J]. Chinese Gardening Abstract. 2012 (9) : 97-2012.

[3] Han Yu. Experience type teaching, the landscape design course teaching practice research [D]. Nanjing: Nanjing College of Art. 2012

[4] Xiang Xiaoxiao. Environmental art professional teaching reform of landscape design study [J]. Journal of Nanchang Institute of Education. 2013 (4)

[5] liu Chun-bo . Analyses of landscape environmental art [J]. The Young Writer. 2010 (18) 